\title{
Computer Vision and Image Processing in Postal Automation
}

\author{
G,Garibotto, C.Scagliola \\ Elsag Bailey, Genova, Italy \\ email: giovanni.garibotto@elsag.it
}

\begin{abstract}
The paper is intended to provide an overview of Computer Vision applications within the context of Postal Automation services. The main functional requirements are briefly referred, as well as the involved Vision functions, which are considered here in a broad sense, including Pattern Recognition, Image Processing and understanding, Signal Processing and Robot Vision. New trends as well as new services emerging in Postal Automation are also discussed, trying to highlight the expected impact on the development of Computer Vision technology.
\end{abstract}

\section{Introduction}

Mail sorting and postal automation has always represented an important area of application for Image Processing and Pattern Recognition techniques. Around the mid of the seventies, the escalating use of faxes and data transfer, and more recently of e-mail, led to predictions that within 20 years relatively few people would communicate by letter. In spite of such predictions mail volume grows steadily, to reach such levels of about 5.6 billion pieces a year in Italy in 1994 [1], 10.3 billion in France in 1994, 10.6 billion in Canada in 1992/1993 [3], and 181 billion in the USA in 1995 [4]. However. the scenario of postal services is rapidly changing all around the world, mainly for the telematics revolution that enables software developers to offer alternate services in competition with traditional providers. Moreover, the global market rules force to break the national monopolies in communication and mail services, also encouraging the information exchange among all providers. As a final result, the new situation is such that Postal Administrations are pressed by a strong competition. Many of the foreseen improvements and new services are based on Image Processing and Computer Vision functions, as will be described later in this report. The following is devoted to the description of the present situation and trends in postal mechanisation and mail processing. New image-based functions are also examined to provide a further improvement of the postal service as well as to introduce new services to the customers.

\section{Postal Mechanisation and Mail Processing}

Mail-handling is a very labour intensive process and labour costs have been increasing during the last three decades. Mail has to be sorted for a large number of destinations. In the US, the national delivery network reaches nearly 128 million addresses [4]. For important destinations like large cities, direct bundles are formed, but for small villages mail is combined into bundles and dispatched to regional sorting centres for further inward sorting. 
The tendency of most Postal Administrations is the introduction of new postcode containing information which could be used for the entire mail-handling process up to the final delivery point.

Sorting is usually performed by machines that read a barcode on the envelope, that identifies the mail piece destination. The barcode is impressed on the envelope by an "encoding" function, performed either automatically by a postal OCR machine or by an employee through a videocoding station. Traditionally, encoding mirrors sorting: a first encoding step identifies the destination city for outward sorting, by reading the classical postcode. The second encoding step identifies the final delivery point for inward sorting, by reading the new, complete, postcode (where it exists and when the user has written it on the address) or by reading the complete address, i.e. street name and number and possibly apartment number, or Post Office Box number, or the name of a large customer, like a Bank or a Company.

In order to reduce the cost of this encoding function, the tendency of Postal Administrations is to gradually increase the percent of mail that is encoded automatically, and to perform this operation only once, i.e. to encode mail to the destination point directly in the first sorting centre. This operation, that requires the on-line consultation of a nation-wide address database, can be already done for a very large proportion of typewritten mail, but a goal of most Postal Administrations is to encode automatically to the destination point also hand-written mail.

The flow-chart of fig 1 refers a network architecture proposed to manage in a uniform way all information and sharing the appropriate resources for the mail sorting process.

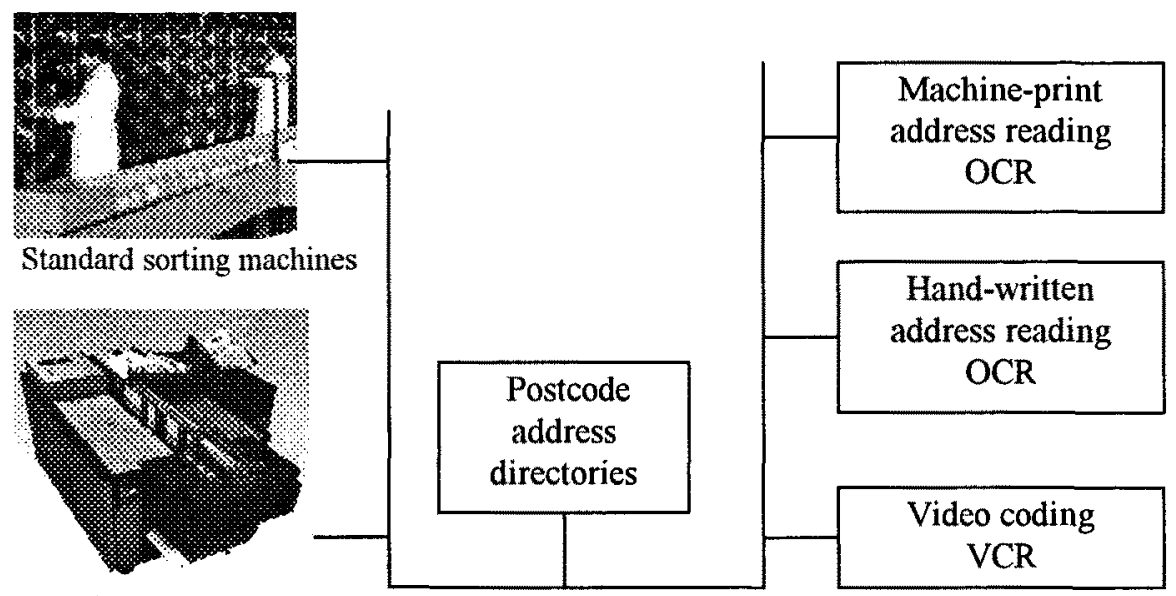

Special sorting machines

Fig. I Flow chart of a network service for mail handling 


\subsection{Mail Processing; statement of the problem and application requirements.}

Automatic reading is necessary for all the address fields necessary for the carrier to the final destination. The current mail flow is roughly sketched in fig.2, from the collection of all mail items to the first office. All items are singulated (culling), oriented and packed together (facing and cancelling), and are sorted according to the post code. At the delivery, destination office, two further sorting process are implemented in order to obtain the final carrier sequencing of the mail.

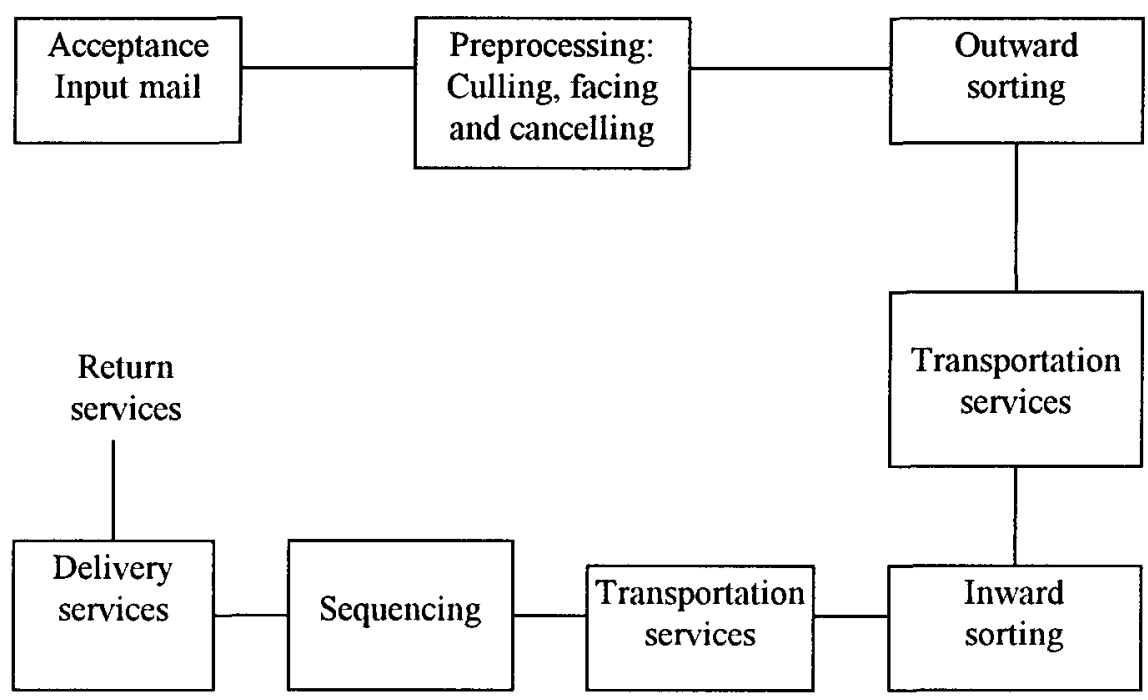

Fig.2 Current mail flow

\subsection{Functional Architecture of a postal address reader}

In a very schematic way, address reading may be described as a data compression process, from the raw data coming at $8 \mathrm{pixel} / \mathrm{mm}$ and 256 grey levels. (something about 2 Mbytes data) down to a few bytes, corresponding to the content of the postal destination address.

It consists essentially of 3 modules, acquisition and Image Processing, segmentation and character recognition and ontext analysis and address recognition.

The input of the recognition phase is the bit-map obtained from the letter image. The firt processing step consists in localisation of text lines (both in the case of handwritten or typewritten text) as well as other geometric or pictorial features to be detected in the mail item (stamp, codes, etc.)

From the localised block of text it is necessary to segment the individual characters for the following recognition. Main problems here are the correct segmentation of broken and touching characters, especially when dealing with hand-written text (both numerals and alphabetic characters). 
Finally character recognition is performed and the literature on this subject is extremely wide and rich [5], including the use of feature based statistical approaches, a variety of pattern matching schemes and a combination of neural network techniques. There has been always a great interest to establish evaluation criteria and benchmarking procedures, to help a quantitative objective comparison of such a wide range of solutions and implementation techniques. The US National Institute of Standards (NIST) has organised specific conferences [6] to promote a thorough comparison of results on the basis of selected data bases representative of machine print and hand print text.

\section{Hand-written address reading}

The new frontiers in handwriting recognition make extensive use of the context to achieve unconstrained address reading, using both large vocabulary and grammar constraints as well as heuristics. A first short-term objective consists in reading the last line including the postcode, city name and state and integrate such information in order to improve the reliability of the system and minimise the use of off-line video coding. The French Postal Administration expects a $10 \%$ increase in automatically sorted (outward sorting) hand-written mail, by integrating the reading of the city name with that of the postcode.

Next generation machines will include also the capability to read the full handwritten address line, with street name and civic number, in order to manage the final postman's delivery. The goal of USPS, the United States Postal Service, is to encode to the delivery point $50 \%$ of the hand-written mail, with $1 \%$ error rate. Laboratory tests indicate that this goal can be achieved [7].

\section{Address Block Location and recognition in Flats sorting machines}

Flats reading represent one of the most challenging objective for postal sorting machines, due to their complexity and the large variety of object categoies which have to be managed by the system. The most common class of flats includes large A4 size envelopes with more or less additional information printed on it (sender and destination address, advertisement messages, stamps and mail class service information). Another class consists of journals, newspapers and catalogues with or without plastic cover. Moreover in the flat category are often included also small parcels with a maximum thickness of about $40 \mathrm{~mm}$.

Only very few flat sorting machines are currently in operation, often without automatic reading capability, but the traffic for this kind of mail is constantly growing, (approximately $21 \%$ of regular normal mail are flats) and the need for automatic encoding is emerging rapidly.

However, automatic reading of addresses on flats cannot be achieved through a simple reengineering of a letter reader. The different characteristics of the mailpieces pose different problems to the image acquisition subsystem and to the processing algorithms, that are not yet fully satisfactory in unconstrained operating conditions and require more sophisticated Image processing and Computer Vision capabilities. 
One of the main problems comes from the management of plastic cover which prevents a sharp and well contrasted image acquisition from the input vision sensor. Improvements in the acquisition process (both in resolution and dynamic range) as well as adaptive grey level image processing tools, represent key factors to solve this problem.

The second critical point is Address Block Location (in short ABL). In fact, in some cases, like magazines and advertisements, the destination address is usually written on a small label floating under a plastic cover, which means that it may be found in any position and with any orientation. In other cases the destination address has to be located in a complex image, full of text and graphics, as newspapers, where the statistical properties of the address block are very close to those of the full size image. The input image data are of size $2000 \times 3000$ pixels or more. The main steps of an $\mathrm{ABL}$ are pre-processing and noise removal, Multiresolution Region of Interest analysis, Segmentation of block candidates and finally Context analysis to classify the detected blocks and decide on their arrangement onto the mail piece.

Many research results on this subject, as well on the other topics of mail process automation can be found on the proceedings of the USPS Conference [9]

\section{Parcel classification}

Huge and complex machines are currently used for 3D parcel sorting. State of the art sorting equipment can handle some 250,000 items per day.

Presently parcel processing is highly labour intensive: in fact parcels are introduced manually by human operators and during this input stage a preliminar selection is already performed according to their size and shape (roll and cylinders, regular and irregular packets, etc.). They are also labelled with some ID code label and the operators place the parcel item on the sorter tray with the label side facing up. At the input stage an overhead scanner is installed to automatically read the label and assign the destination information to the sorter tray.

During the last 10 years there has been a significant research effort carried out by the most important Postal Administration, to automatize the parcel input stage and reduce the cost associated to this very low-professional work. The task here is $3 \mathrm{D}$ object classification of the parcel item into one of the three main categories (cylindrical items, thick and almost polyhedral parcels, irregular shapes).

3D parcel reconstruction has been a hot research topics since many years in the scientific community of Computer Vision. It is possible to classify the reconstruction techniques in two main classes. The first one is based on passive vision (stereovision and motion analysis) and the second one is based on the geometric analysis of projected light patterns (lasers or white light projectors).

Actually a successful use of Computer Vision requires a deep reorganisation of the whole handling and sorting approach and this may be the reason for the presently limited success of automatic parcel classification. Main are reliability problems of current solutionsand the requirements of flexibility when no a-priori manual ID-label application is performed. Moreover this classification is often not sufficient to fully automatize the parcel input stage. A human operator is required anyway to place the ID-label and orient the parcel with such label facing-up. 


\section{Material Handling and station loading/unloading}

One of the most intensive tasks to be performed in a large mail distribution centre is the transport of mail items between different machines and from/to the input/output stage of the centre.

The loading/unloading of the letters to/from mail sorting machines is still mainly performed by human operators and the automation of this process represents an essential function to achieve. A commonly agreed approach consists in the realisation of standardised letter containers which could be handled automatically by robotic machines.

From recent studies it has been demonstrated that such transport service is carried out for about $\mathbf{8 0} \%$ through the use of trolleys pushed by human operators and just $20 \%$ is managed by electric trucks (again manually driven).

The available technologies to solve such problem of transportation between different working cells (intercell service) are:

- Electrical trucks, often used to tow a convoy of passive trolleys, with obvious problems of manoeuvrability and requirement of human driving.

- Rail transport system with the well known disadvantages of fixed installations and no flexibility in the management of the mail distribution centre.

- Rollers chains are efficiently used for point-to-point service, but again there are strong limitations due to space occupancy and no flexibility.

- The use of AGVs (Autonomous Guided Vehicles) represents nowadays the most flexible solution. There are conventional systems using inductive guides buried into the floor, as well as new generation navigation systems, mainly based on active sensors (magnetic tags or lasers) for self-orientation. Still there is poor flexibility in the reconfiguration of the navigation map, high precision requirements in the positioning of the loads (pallets), difficulty to switch from automatic to manual driving of the vehicle.

New generation mobile robots, based on advanced sensor, with free ranging capabilities, with the possibility of an easy reconfiguration of the navigation route, able to detect the presence of other vehicles at crossing points, with automatic and manual driving capability, represent the expected solution.

In this domain Computer Vision may play a fundamental role to give flexibility and intelligence to the robotic logistic system.

\subsection{Computer Vision for Autonomous navigation}

There are not many industrial experiences of Computer Vision applied to AGV's even if this is commonly considered one of the most promising solutions to achieve the necessary flexibility. On the other hand, there are interesting vision-based control techniques which have been developed in the automotive industry as a support to the driver [10]. Autonomous navigation has been strongly pushed by military research for both normal road drive and off-road navigation. There has been also attempts to use Computer Vision to drive AGV systems and in mobile robotics for service applications, as in [13] for hospital transportation functions. More recently, a fully passive vision approach has been proposed to allow self-positioning and autonomous navigation of a fork-lift carrier named Robolift, [14]. In this case Computer Vision is 
used to recognise artificial geometrical landmarks, placed along the navigation pathway and correct the relative odometry estimates. Furthermore, Computer Vision is also used for docking control, to recognise the correct position of the pallet to optimize the displacement of the fork prongs before loading.

\section{New added value services in Postal Automation}

Today large volume mailers increasingly demand faster, more reliable service and customised products. They want day-certain delivery, shipment and piece tracking, and an electronic data interface. Moreover, the most important administrations now compete with express mail service companies like DHL, UPS, FedEX, newspapers, telecommunications companies, and alternative delivery services.

New functions are under investigation to enhance the level of postal service to the final customer.

- verification of the presence and value of stamp(s) and postage, with reference to weight, destination and class of the postal object;

- detection of false or recycled stamps;

- identification of special stamps and logos for "controlled delivery by time";

- reading of the amount of additional stamps for effecting payments;

- reading and verification of postal permits;

- reading the name of the addressee for redirection services;

- reading the address of the sender for "return to sender" mail.

\subsection{Paper to Electronic mail service.}

The mail process has been primarily considered as an end-to-end paper mail service. On the other hand, the integration of different technologies can provide excellent opportunities for new postal services as hybrid mail, as an example of electronic to paper mail service. In this case large mailers can produce and forward messages in electronic form and use the distributed postal network for the printing and delivery of the mail.

Image-based technologies make it possible now to cross the paper/electronic barrier in the opposite direction and implement paper-to-electronic services.

An example of a new service of this type is the Reply Card Processing, or automation of Business Reply Mail. This service allows the interception of all business reply cards or courtesy reply cards addressed to a specific customer (i.e. a mail order company) and the capture of the image on both sides of the card. At this point, the image can be stored in appropriate electronic mail boxes and later transmitted to the customer's fulfillment centre instead of the physical card. This paper-to-image transformation alone can reduce the delivery time from 2 to 4 days down to 12 to 18 hours [16] and it is the first step foreseen for a new service in the USA [4].

\section{Conclusions}

The mail automation sector is a quickly evolving area of industrial automation, where the development of Electronics and Parallel processing, Sensors, Robotics, 
Information technology and Telecommunications, has opened new perspectives of development.

In the last twenty years the Postal sector has been a very closed domain with special purpose solutions and approaches and a poor connection with near applications and research disciplines. The mail sorting process has been, and it is, heavily dependent by mechanical constraints. It is always an exciting experience to visit a Mail sorting and Distribution Centre, with huge and complex machines and thousands of letters running back and forth at incredible high speed along the rubber transport chains. The other foremost technology has been definitely OCR for address reading.

But now there are new problems and new solutions emerging, as shortly discussed in this paper. The reading process is much more complex than measuring accuracy or speed of the individual character recognition module. The intelligence to distinguish the different pieces of information in the mail (background, form structures, etc.), the possibility to take advantage of all available context information to drive the OCR and text reading, with feedback control, is the real challenge of new generation reading systems.

Finally 3D Computer Vision appears to become an important enabling technology in the Postal Automation sector, being mainly focused to support robotic applications in material handling and logistics services.

\section{References}

[1] Poste Italiane, "Volume Traffico Nazionale", World Wide Web site: http://www.nettuno.it/fiera/posteitaliane/roma/html/4bc-corr.gif

[2] Canada Post Corporation, "Progress Report", 11-th Int. Conference on Postal Mechanisation, Melbourne, March 7-11, 1994.

[3] United States Postal Service, "Postal Facts for Fiscal Year 1995", World Wide Web site: http://www.usps.gov/history/pfact95f.htm.

[4] Proceedings of the Third International Conference on Document Analysis and Recognition, IEEE Computer Society Press, Aug. 1995.

[5] "The First Census Optical Character Recognition System Conference", NISTIR 4912, Aug. 1992, U.S. Dep. of Commerce, NIST.

[6] F.Kimura \& M.Shridhar, "Hand-written Address Interpretation using Extended Lexicon Word Matching", 5-th Int. Workshop on Frontiers in Handwriting Recognition, Colchester, September 2-5, 1996.

[7] Proceedings of the Advanced Technology Conference, USPS, 1992,

[8] B.Ulmer, "VITA II - Active Collision Avoidance in Real Traffic", IEEE Symposium on Intelligent Vehicles '94, Oct. 1994, Paris, pp. 1- 6.

[9] E.D.Dickmanns, R.Behringer, D.Dickmanns, T.Hildebrandt, M.Maurer, F.Thomanek, J.Schielen, "The Seeing Passenger Car VaMoRs-P", IEEE Symposium on Intelligent Vehicles '94, Oct. 1994, Paris, pp. 68- 73.

[10] J.M.Evans, "HelpMate: a service robot success story", Service Robot: An International Journal, Vol 1 n.1, 1995, pp.19-21, MCB University Press.

[11] G.Garibotto, "ROBOLIFT: vision guided autonomous fork-lift", Service Robot: An International Journal, Vol 2 n.3, 1996, pp.31-36, MCB University Press. 